\title{
Forschung in der Grundversorgung?
}

Die Grundversorgung als Spezialität existiert in der Schweiz offiziell seit 25 Jahren - Datum der Gründung der Schweizerischen Gesellschaft für Allgemeinmedizin (SGAM) -, und im Jahre 2002 wurde auf europäischer Ebene eine gemeinsame Definition des «Allgemeinmediziners» bzw. des «Hausarztes» festgelegt. Die Lehre im Bereich Allgemeinmedizin wurde 1980 durch einen Beschluss des Parlamentes eingeführt; wo aber ist die zweite Säule einer Disziplin, die sich als akademisch versteht: die Forschung?

Weder die Einsetzung einer Arbeitsgruppe «Forschung» der SGAM im Jahre 1981 (zu der sich später eine weitere des Kollegiums für Hausarztmedizin gesellte), noch die frommen Wünsche anlässlich eines SAMW-Seminars über klinische Forschung vor 10 Jahren [1] haben dazu geführt, dass in diesen Bereich richtig Bewegung gekommen ist. Dafür gibt es verschiedene Gründe: das Fehlen wirklicher und schweizweit anerkannter akademischer Institutionen trotz vielversprechender Entwicklungen in Bern (FIAM) und in Lausanne und Genf (Gründung von «Départements de Médecine communautaire»); die Tatsache, dass sich drei Gesellschaften den Bereich der Grundversorgung teilen (und teilweise neiden) - dies trotz der lobenswerten Bemühungen, die Kräfte auf der Ebene des Kollegiums zu bündeln; die Schwierigkeit, die wissenschaftliche Basis der Grundversorgung zu definieren; das Fehlen entsprechend ausgebildeter Kader; und noch vieles andere.

Trotzdem ist all dies kein Grund, eine festliche Trauerrede auf die Forschung in der Grundversorgung zu halten [2]. Ein von der SAMW organisiertes Seminar, welches Mitte November 2002 in Basel interessierte Fachleute vereinigte (mit Vertretern der drei im Bereich Grundversorgung tätigen Fachgesellschaften, des Kollegiums, der Fakultäten und der SAMW), hat das Gegenteil bewiesen: Tatsächlich existiert ein ausgesprochener Wille, im Bereich Forschung in der Grundversorgung vorwärtszumachen. Referenten aus dem Ausland (Prof. A. Knottnerus aus Maastricht in den Niederlanden und Prof.
F. Olesen aus Aarhus in Dänemark) haben gezeigt, dass Länder von vergleichbarer Grösse hier durchaus erfolgreich sind; in den anschliessenden Workshops fehlte es auch nicht an Ideen, in welche Richtungen man aktiv werden müsste:

- Sensibilisierung von Medizinstudierenden für die Forschung in der Grundversorgung («summer school»?);

- Organisation eines Masterangebotes «Forschung in der Grundversorgung» auf schweizerischer Ebene (ähnlich dem Master in Public Health);

- Fortbildungsangebote für praktizierende Ärzte, welche sich an der Forschung beteiligen wollen (ähnlich den Sentinella-Gruppen);

- Ad-hoc-Bildung von Netzwerken (Typ «Sentinella») für klar definierte Projekte;

- Sensibilisierung von Politikerinnen und Politikern, Kassenverantwortlichen und Sponsorinnen und Sponsoren für diesen vielversprechenden Bereich.

Am Ende der Tagung vom 15. November 2002, über welche Bruno Kissling im PrimaryCare umfassend berichten wird [3], waren sich alle einig: Diesmal müssen wir vom Enthusiasmus, welchen die Referate und Diskussionen ausgelöst haben, profitieren; diesmal müssen wir handeln, bevor diese einzigartige Gelegenheit der Einigkeit aller Teilnehmerinnen und -nehmer verstreichen lassen: die Grundversorgung ist eine akademische Disziplin, welche eine hochkarätige Forschung verdient!

Prof. Hans Stalder, Genf Präsident der Kommission «Recherche et réalisation en médecine appliquée» der SAMW

1 Stalder H. La recherche en médecine ambulatoire. Schweiz Ärztezeitung 1993;74:1124-6.

2 Künzi B. 20 Jahre Forschung der SGAM eine festliche Trauerrede. www.sgam.ch

3 Kissling B. Förderung der Forschung in ambulanter Medizin. PrimaryCare 2003;3(5):55-9. www.primary-care.ch. 